\title{
Complex Ordered Patterns in Mechanical Instability Induced Geometrically Frustrated Triangular Cellular Structures
}

\section{Citation}

Kang, Sung Hoon, Sicong Shan, Andrej Košmrlj, Wim L. Noorduin, Samuel Shian, James C. Weaver, David R. Clarke, and Katia Bertoldi. 2014. Complex Ordered Patterns in Mechanical Instability Induced Geometrically Frustrated Triangular Cellular Structures. Physical Review Letters 112(9): 098701.

\section{Published Version}

doi:10.1103/physrevlett.112.098701

\section{Permanent link}

http://nrs.harvard.edu/urn-3:HUL.InstRepos:12111385

\section{Terms of Use}

This article was downloaded from Harvard University's DASH repository, and is made available under the terms and conditions applicable to Other Posted Material, as set forth at http:// nrs.harvard.edu/urn-3:HUL.InstRepos:dash.current.terms-of-use\#LAA

\section{Share Your Story}

The Harvard community has made this article openly available.

Please share how this access benefits you. Submit a story.

Accessibility 


\title{
Complex Ordered Patterns in Mechanical Instability Induced Geometrically Frustrated Triangular Cellular Structures
}

\author{
Sung Hoon Kang, ${ }^{1}$ Sicong Shan, ${ }^{1}$ Andrej Košmrlj, ${ }^{2}$ Wim L. Noorduin, ${ }^{1}$ Samuel Shian, ${ }^{1}$ \\ James C. Weaver, ${ }^{3}$ David R. Clarke, ${ }^{1}$ and Katia Bertoldi ${ }^{1,4, *}$ \\ ${ }^{1}$ School of Engineering and Applied Sciences, Harvard University, Cambridge, Massachusetts 02138, USA \\ ${ }^{2}$ Department of Physics, Harvard University, Cambridge, Massachusetts 02138, USA \\ ${ }^{3}$ Wyss Institute for Biologically Inspired Engineering, Harvard University, Cambridge, Massachusetts 02138, USA \\ ${ }^{4}$ Kavli Institute, Harvard University, Cambridge, Massachusetts 02138, USA
}

(Received 5 December 2013; published 5 March 2014)

\begin{abstract}
Geometrical frustration arises when a local order cannot propagate throughout the space because of geometrical constraints. This phenomenon plays a major role in many systems leading to disordered ground-state configurations. Here, we report a theoretical and experimental study on the behavior of buckling-induced geometrically frustrated triangular cellular structures. To our surprise, we find that buckling induces complex ordered patterns which can be tuned by controlling the porosity of the structures. Our analysis reveals that the connected geometry of the cellular structure plays a crucial role in the generation of ordered states in this frustrated system.
\end{abstract}

DOI: 10.1103/PhysRevLett.112.098701

A geometrically frustrated system cannot simultaneously minimize all interactions because of geometric constraints $[1,2]$. The origin of this phenomenon can be easily illustrated by looking at the arrangement of spins with antiferromagnetic interactions on a triangle. In contrast to the case of a square, each spin on a triangle cannot be antialigned with all of its neighbors [see Fig. 1(a)]. Therefore, the system is frustrated and is characterized by degenerate ground states [3]. Given the difficulty of probing individual spin states in a system without disrupting their states [4], a number of artificial frustrated systems with individual discrete elements that can be directly monitored have been investigated to understand how spins accommodate the frustration of their interactions. These include artificial spin ice systems [4-11], colloid systems $[2,12,13]$, and periodically arranged magnetic rotors [14]. Since geometrical frustration typically gives rise to disordered configurations, there has been growing interest in investigating mechanisms to generate order in frustrated systems. Interestingly, it has been reported that ordered configurations can be achieved either by introducing long-range interactions or lifting the geometrical constraints. In fact, the ordering of both pyrochlore magnets [15] such as $\mathrm{Ho}_{2} \mathrm{Ti}_{2} \mathrm{O}_{7}$ and $\mathrm{Dy}_{2} \mathrm{Ti}_{2} \mathrm{O}_{7}$ and artificial spin ice [11] is attributed to long-range dipolar interactions $[3,16,17]$. In addition, ordered configurations have been achieved by introducing dopants or defects $[18,19]$ and distorting the lattice $[2,20,21]$ to relax the geometrical constraints.

Here, we report a system consisting of elastic beams connected to form a two-dimensional triangular lattice in which a simple and ubiquitous phenomenon such as buckling [22] induces geometrical frustration. The essence
PACS numbers: 89.75.Kd, 46.32.+x, 46.70.-p, 62.20.-x

of this phenomenon can be easily captured by comparing a square and a triangular frame, as shown in Fig. 1(b). In the unfrustrated square frame each beam can buckle into the most energetically favored configuration-a half sinusoid-and simultaneously preserve the angles with all its neighbors at the joints to minimize the deformation energy. On a triangular frame, however, such configurations are impossible, so the system becomes frustrated. Then, what kind of patterns induced by buckling would appear in a triangular cellular structure? Interestingly, we show both numerically and experimentally that buckling in frustrated triangular cellular structures results in the formation of complex ordered patterns.

To understand patterns emerging as the result of buckling in a triangular cellular structure, we began by analytically (a)

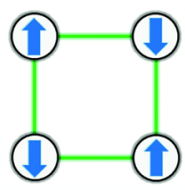

(b)

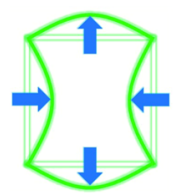

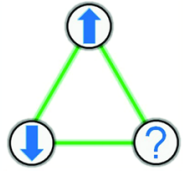

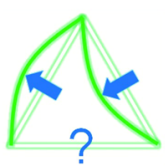

FIG. 1 (color online). Geometrical frustration. (a) In antiferromagnetic systems nearest neighbor spins want to align in opposite directions. This rule can be easily satisfied on a square. However, due to geometrical frustration it is not possible to satisfy it on a triangle. (b) Similarly, buckled beams on frames want to preserve angles at joints to minimize the deformation energy. Again this can be realized for square frames, but not for frustrated triangular frames. 
investigating the stability of a single triangular frame consisting of three elastic beams of bending stiffness $E I$ and length $L$, rigidly connected to each other, so that their relative orientation was fixed. Assuming that the beams were slender so that the contribution of the shear forces could be neglected, we used the stiffness matrix of a beam column [23] and imposed equilibrium conditions at each joint to determine the critical load and the corresponding modes (see Supplemental Material [24] for details). The analysis revealed that if all the three beams experienced the same compressive force $P$, the frame buckles as early as $P=14.87\left(E I / L^{2}\right)$, leading to the formation of patterns for which

$$
\theta_{A}+\theta_{B}+\theta_{C}=0,
$$

where $\theta_{A}, \theta_{B}$, and $\theta_{C}$ denote the rotation of the three junctions [see Fig. 2(a)]. Therefore, this simple analysis confirmed that buckling introduces degeneracy, since there are an infinite number of modes satisfying Eq. (1), ranging from a symmetric pattern with $\theta_{B}=0$ and $\theta_{A}=-\theta_{C}$ to a chiral configuration with $\theta_{A}=-2 \theta_{C}$ and $\theta_{B}=\theta_{C}$ [see Fig. 2(a) and Fig. S3].

We next performed finite element (FE) simulations to investigate the patterns induced by buckling in finite size periodic triangular cellular structure. We built twodimensional models consisting of different numbers of unit cells [here we considered a unit cell composed of two adjacent triangles, see Fig. 2(c)] and investigated the stability of the system under equibiaxial compression using the commercial finite element package ABAQUS. We used a linear perturbation procedure to predict the critical strains (eigenvalues) associated with various buckled configurations (eigenmodes) and we expected the observed buckled configuration to be the one with the smallest critical strain. We find that the system-independent of its size and porosity - is characterized by two eigenmodes with very close critical strains [the critical strain difference is less than $0.1 \%$ for the $11 \times 12$ unit cells shown in Fig. 2(b). See also Tables S1-S3 in the Supplemental Material [24]]. Interestingly, if we focus on the center part of the models, which is only minimally influenced by edge effects, we see that both eigenmodes are characterized by ordered patterns with a periodic unit consisting of $3 \times 3$ unit cells, as shown in Fig. 2(b) for a structure with $11 \times 12$ unit cells.

Recognizing that the finite-sized specimens are necessarily influenced by boundary conditions at the edges, we also investigated the stability of infinite periodic triangular lattices. Since buckling may alter the periodicity of the structure, we considered super cells consisting of $m \times n$ undeformed unit cells subjected to periodic boundary conditions and calculated the critical strain for each of them. The critical strain of the infinite periodic structure is then defined as the minimum critical strain on all considered super cells. The results confirmed those of the finite (a)

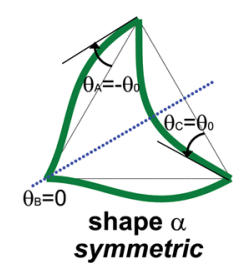

(b)

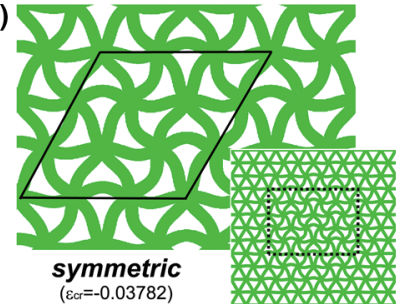

(c)

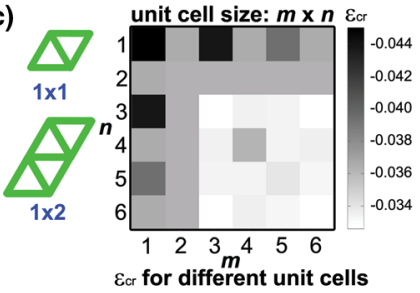

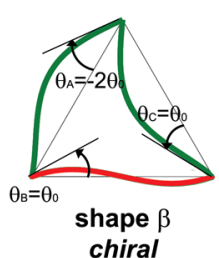

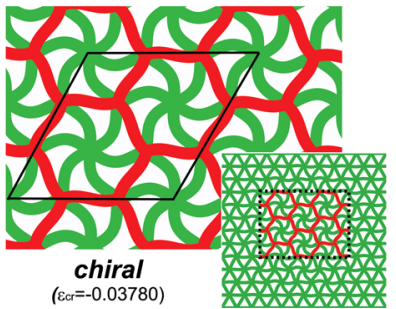

(d)

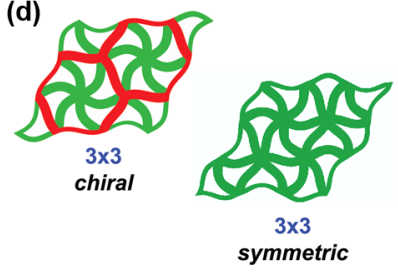

FIG. 2 (color online). Analytical and numerical modeling. (a) Representative critical eigenmodes obtained from the buckling analysis of a single triangular frame. The dotted line indicates the mirror plane for the symmetric pattern. The beam that buckles into a full sinusoid is indicated in red. (b) First and second eigenmodes obtained from the finite element analysis for a lattice structure comprising $11 \times 12$ unit cells under equibiaxial compression. The frame is constructed from beams with thickness $(t)$ to length $(L)$ ratio of 0.2 . Note that the eigenvalues associated with the two modes are very close to each other. Both the full structures and the magnified views of their central regions are shown. (c) Critical strain for super cells with $t / L=0.2$ consisting of $m \times n$ unit cells, where a unit cell consists of two adjacent triangles. In the analysis, we used periodic boundary conditions to eliminate edge effects. The results indicate that the $3 \times 3$ configuration has the minimum critical strain. (d) Two buckling patterns (one symmetric and one chiral) are associated with the critical strain of the $3 \times 3$ configuration. For the chiral pattern, the mirror image of the pattern can also appear.

size simulations and showed that the $3 \times 3$ configuration has the minimum critical strain with two possible eigenmodes [Figs. 2(c) and 2(d) and Tables S4 and S5].

To investigate the origin of the ordered patterns induced by buckling in our frustrated system, we introduced a spinlike model. Our goal is to construct a simple model for estimating the energy associated with different spin configurations corresponding to buckled beams, and for identifying the minimum energy configurations. Since in two dimensions a beam can buckle "up" or "down," we represent its buckling direction with an Ising-like unit spin vector $\mathbf{s}_{i}$ [see Fig. 3(a)]. Such spins are placed at the centers of prebuckled beams, forming the well-known kagome lattice $[25,26]$. For the sake of simplicity, we first consider 
the case where every beam buckles into a half sinusoid so that the energy difference between configurations comes from changes in relative orientation at the joints (i.e., at triangle corners). Here, the deformation of the $\pi / 3$ angle between neighboring beams is associated with the energy cost $J_{1}>0$. Therefore, the total energy of a buckled configuration is given by

$$
E=-\sum_{\langle i, j\rangle} J_{1}\left(\mathbf{s}_{i} \cdot \mathbf{s}_{j}-1 / 2\right),
$$

where $\mathbf{s}_{i}$ and $\mathbf{s}_{j}$ are two nearest neighbor spins [e.g., purple arrows with respect to the reference spin in Fig. 3(a)]. Such antiferromagnetic spin model on the kagome lattice has been studied extensively $[25,26]$. For a structure comprising $N$ triangles, the minimum energy configurations correspond to a tiling with $N$ buckled units $\alpha$ from Fig. 2(a) $\left(E_{\min }=N J_{1}\right)$, and it has been shown that there are exponentially many ground states due to geometric frustration $[25,26]$. Therefore, we find exponentially many disordered tilings [e.g., see Fig. 3(b)] and only a small number of ordered tilings, one of which corresponds to the symmetric ordered pattern observed in the numerical analysis [Fig. 3(c)].

However, the finite element simulations described above are favoring ordered structures. The reason for this might be interactions among neighboring triangles coupled through joints. To take this into account, we consider the second nearest neighbor interactions between spins [see Fig. 3(a)] and assign an energy cost $J_{2}\left(0<J_{2} \ll J_{1}\right)$ to connected beams that deform the $2 \pi / 3$ angle. For simplicity, we assume that the energy costs of deformations of $\pi / 3$ and $2 \pi / 3$ angles at joints are additive, so that

$$
E=-\sum_{\langle i, j\rangle} J_{1}\left(\mathbf{s}_{i} \cdot \mathbf{s}_{j}-1 / 2\right)+\sum_{\langle k, l\rangle} J_{2}\left(\mathbf{s}_{k} \cdot \mathbf{s}_{l}+1 / 2\right),
$$

where $\mathbf{s}_{k}$ and $\mathbf{s}_{l}$ are pairs of second nearest neighbor spins [e.g., blue arrows with respect to the reference spin in Fig. 3(a)] and $J_{1}$ and $J_{2}$ are positive quantities, since any elastic deformation is associated with a positive energy cost. This additional interaction breaks the degeneracy, so that the symmetric ordered configuration shown in Fig. 3(c) is the only one that minimizes the energy given in Eq. (3) [26]. Our findings nicely agree with previous observations reported for antiferromagnetic systems [7] where additional interactions (often long ranged) are needed for ordered ground states to emerge. Moreover, it is worth noting that for this ordered symmetric pattern we find a striking correlation between its arrangement of spins and that of the ideal spin solid [9].

The spinlike model described above can also be generalized to allow for a second buckling mode of the beams, which is associated with the energy cost $\Delta$. This is achieved by assigning two spins to each beam, where aligned (a)

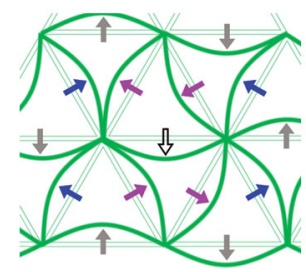

$\Rightarrow$ reference spin

$\rightarrow$ 1st nearest neighbors

$\rightarrow$ 2nd nearest neighbors

$\rightarrow$ negligible contribution

(b)
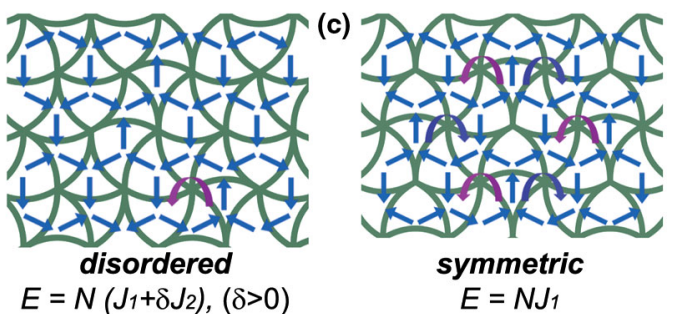

(d)

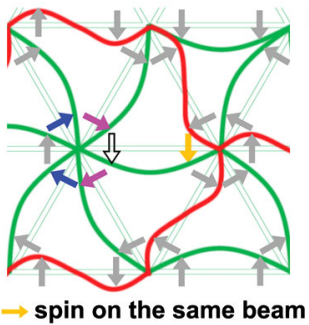

(e)

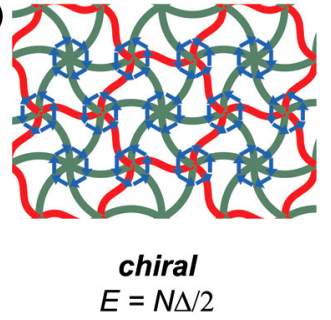

FIG. 3 (color online). Spin-like model. (a) A schematic showing how spins define a buckled pattern when all of the beams buckle into a half sinusoid. (b) Disordered and (c) ordered symmetric configurations predicted by the model. When considering only first nearest neighbor interactions (i.e., $J_{2}=0$ ), there are exponentially many disordered tilings that minimize the energy for a lattice structure with $N$ triangles and only a small number of ordered patterns. However, only the symmetric ordered configuration minimizes the energy if second nearest neighbor interactions are considered (i.e., $J_{2}>0$ ). (d) The spinlike model can be generalized to allow for the second buckling mode of the beams by assigning two spins to each beam. (e) A new chiral ordered state emerges when two spins are assigned to each beam. Again, the mirror image of the chiral pattern can also appear.

and antialigned spins correspond to the first and second buckling mode, respectively [see Fig. 3(d)]. The energy cost of a buckled configuration can then be calculated as

$$
\begin{aligned}
E= & -\sum_{\langle i, j\rangle} J_{1}\left(\mathbf{s}_{i} \cdot \mathbf{s}_{j}-1 / 2\right)+\sum_{\langle k, l\rangle} J_{2}\left(\mathbf{s}_{k} \cdot \mathbf{s}_{l}+1 / 2\right) \\
& -\sum_{\langle m, n\rangle} \frac{\Delta}{2}\left(\mathbf{s}_{m} \cdot \mathbf{s}_{n}-1\right),
\end{aligned}
$$

where $\mathbf{s}_{m}$ and $\mathbf{s}_{n}$ are two spins assigned to the same beam. In this case, in addition to the ordered configuration described above [Fig. 3(c)], a new chiral ordered state emerges [Fig. 3(e)], which can be constructed from tiling the plane with the buckled triangular unit $\beta$ from Fig. 2(a). This chiral configuration preserves both the first and the second nearest angles at joints and is associated with the 
energy $\operatorname{cost} N \Delta / 2$, where the factor $1 / 2$ comes from sharing the second mode buckled beam between two triangles. Thus, when $\Delta / 2>J_{1}$, the minimum energy configuration is the symmetric one [Fig. 3(c)], while when $\Delta / 2<J_{1}$ it is the chiral one [Fig. 3(e)].

Next, we conduct a simple scaling analysis to determine how the parameters $J_{1}, J_{2}$, and $\Delta$ depend on the geometry of the beams (thickness $t$, length $L$, and out-of-plane height $h$ ) and the elastic property of the material (Young's modulus $E$ ). We start by noting that the buckled shape of a thin beam $(t \ll L)$ can be well approximated by [27]

$$
y(s)=A_{m} \sin \left(\frac{m \pi s}{L}\right)
$$

where $m$ is an integer representing the buckling mode and $s$ is the intrinsic length parameter of the beam, $s \in[0, L]$. It follows that $y^{\prime}$ and $y^{\prime \prime}$ scale as

$$
y^{\prime} \sim A_{m} \frac{m}{L}, \quad y^{\prime \prime} \sim A_{m} \frac{m^{2}}{L^{2}} .
$$

Furthermore, the amplitude $A_{m}$ can be related to the small compressive strain $\varepsilon \ll 1$ by considering that the beam is inextensible (i.e., $d s^{2}=d x^{2}+d y^{2}$ ), so that

$$
\begin{aligned}
L(1-\epsilon) & =\int_{0}^{L} d x \\
& =\int_{0}^{L} d s \sqrt{1-\left(y^{\prime}\right)^{2}} \approx L\left(1-\frac{m^{2} \pi^{2} A_{m}^{2}}{4 L^{2}}\right) .
\end{aligned}
$$

By comparing the left and right sides of Eq. (7) we find

$$
A_{m} \sim \sqrt{\epsilon}(L / m)
$$

An estimate for the energy cost $\Delta$ associated with the beam buckling into the second mode can then be obtained using elastic plate theory and approximating the bending energy of the buckled beam as

energy $\sim$ plate area $\times$ bending rigidity $\times\left(\text { curvature } \sim y^{\prime \prime}\right)^{2}$, energy $\sim L h \times E t^{3} \times \frac{\varepsilon m^{2}}{L^{2}}$,

so that

$$
\Delta \sim \epsilon E h t^{3} / L
$$

In a similar way, we can estimate the energy cost $J_{1}$ and $J_{2}$ for deforming the $\pi / 3$ and $2 \pi / 3$ angles between neighboring beams at joints. Since these deformations are localized near joints and only affect a beam region of length $\sim t$, we have
$J_{1}, J_{2} \sim$ volume $\times$ elastic constant $\times\left(\text { local strain } \sim y^{\prime}\right)^{2}$,

$J_{1}, J_{2} \sim h t^{2} \times E \times \epsilon=\epsilon E h t^{2}$.

From the scaling analysis above we find that $\Delta / J_{1}$ scales as $t / L$, indicating the opportunity to tune the formation of the symmetric or chiral pattern by controlling the beam aspect ratio. In fact, we expect that the chiral and symmetric patterns emerge for small and large $t / L$, respectively, and numerical FE simulations predict a sharp first-order-like transition at $t / L \approx 0.24$ [see Table S6]. Finally, it is worth noting that the chiral pattern arises with a new spin configuration, thus, highlighting the richness of our frustrated system.

Guided by our theoretical analysis, we fabricated centimeter scale elastomeric triangular cellular structures with $t / L=0.2$ and $0.3(L=5 \mathrm{~mm})$ comprising $11 \times 12$ unit cells [Figs. 4(a) and 4(b), left]. The experimental results show an excellent agreement with the analytical and numerical predictions. When tested under equibiaxial compression, we, indeed, observed the formation of an ordered chiral pattern for $t / L=0.2$ [Fig. 4(a), center and right]. Moreover, as predicted by the scaling analysis, an ordered symmetric pattern emerged for $t / L=0.3$ [Fig. 4(b), center and right]. Since geometrical frustration in our continuum system is induced by a mechanical instability that is scale independent (where the continuum assumption holds), our findings can be extended to different length scales, materials, and stimuli. In fact, similar patterns were also observed by swelling surface-attached triangular cellular structures [Fig. S5], providing us opportunities to study the effects of kinetics on geometrical frustration. (a)

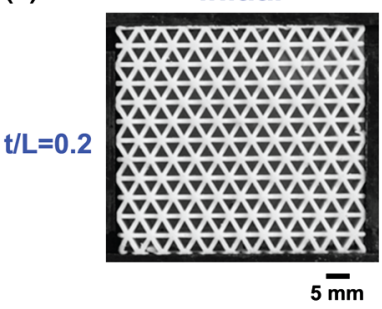

(b)

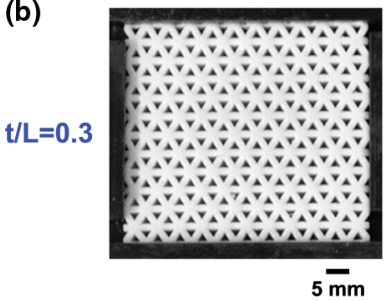

buckled
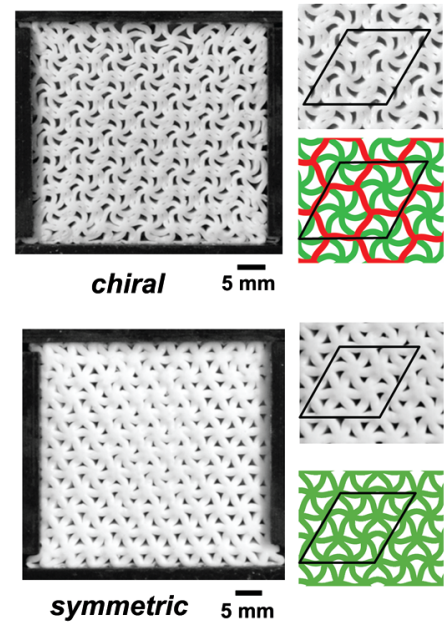

FIG. 4 (color online). Experimental observation of ordered chiral or symmetric patterns. (a), (b) Initial and buckled configurations (at $\epsilon=0.16$ ) for a sample characterized by $t / L=0.2$ and $t / L=0.3(L=5 \mathrm{~mm})$, respectively. The insets on the right show magnified images of the buckled patterns obtained experimentally and numerically. 
In summary, we have studied analytically, numerically, and experimentally geometrical frustration induced by buckling in continuum triangular cellular structures. Interestingly, we found that the connected geometry favors the formation of complex ordered symmetric or chiral patterns, and the appearance of a specific configuration is controlled by the porosity of the system. In stark contrast to most of the artificial frustrated systems previously studied, ordered configurations emerge naturally in our structures, indicating that the coupling between elasticity and geometrical frustration in continuum structures opens the door to a new class of phenomena waiting to be explored. In fact, while the symmetric pattern induced by buckling has a striking correlation with the arrangement of spins in the ideal spin solid, no analogous spin arrangement to that of our chiral configuration has been reported. Our results also indicate that simple changes in the geometry of the elastic structures may lead to novel effects that can be easily visualized, since-unlike for the case of magnetic systems-our experimental system can be quickly fabricated and tested. With their intriguing and rich behavior originating from the interplay between geometry and deformation, continuum geometrically frustrated lattice structures offer many novel phenomena to be investigated.

This work has been supported by Harvard MRSEC through Grant No. DMR-0820484 and by NSF through Grant No. CMMI-1149456 (CAREER) and by the Wyss Institute through the Seed Grant Program. K. B. acknowledges start-up funds from the Harvard School of Engineering and Applied Sciences and the support of the Kavli Institute at Harvard University. The authors are grateful to Professor David Nelson (Harvard University), Professor Joanna Aizenberg (Harvard University), Dr. Elizabeth R. Chen (Harvard University), and Professor Robert Connelly (Cornell University) for helpful discussions.

*Corresponding author. bertoldi@seas.harvard.edu

[1] J.-F. S. Sadoc and R. Mosseri, Geometrical Frustration (Cambridge University Press, Cambridge, England, 2006).

[2] Y. Han, Y. Shokef, A. M. Alsayed, P. Yunker, T. C. Lubensky, and A. G. Yodh, Nature (London) 456, 898 (2008).

[3] L. Balents, Nature (London) 464, 199 (2010).

[4] R. F. Wang, C. Nisoli, R. S. Freitas, J. Li, W. McConville, B. J. Cooley, M. S. Lund, N. Samarth, C. Leighton, V. H. Crespi, and P. Schiffer, Nature (London) 439, 303 (2006).
[5] Y. Qi, T. Brintlinger, and J. Cumings, Phys. Rev. B 77, 094418 (2008).

[6] P. E. Lammert, X. Ke, J. Li, M. Khan, C. Nisoli, D. M. Garand, V. H. Crespi, and P. Schiffer, Nat. Phys. 6, 786 (2010).

[7] S. A. Daunheimer, O. Petrova, O. Tchernyshyov, and J. Cumings, Phys. Rev. Lett. 107, 167201 (2011).

[8] J. P. Morgan, A. Stein, S. Langridge, and C. H. Marrows, Nat. Phys. 7, 75 (2011).

[9] W. R. Branford, S. Ladak, D. E. Read, K. Zeissler, and L. F. Cohen, Science 335, 1597 (2012).

[10] A. Farhan, P. M. Derlet, A. Kleibert, A. Balan, R. V. Chopdekar, M. Wyss, L. Anghinolfi, F. Nolting, and L. J. Heyderman, Nat. Phys. 9, 375 (2013).

[11] S. Zhang, I. Gilbert, C. Nisoli, G.-W. Chern, M. J. Erickson, L. O'Brien, C. Leighton, P. E. Lammert, V. H. Crespi, and P. Schiffer, Nature (London) 500, 553 (2013).

[12] Y. Shokef and T. C. Lubensky, Phys. Rev. Lett. 102, 048303 (2009).

[13] P. Yunker, Z. Zhang, and A. G. Yodh, Phys. Rev. Lett. 104, 015701 (2010).

[14] P. Mellado, A. Concha, and L. Mahadevan, Phys. Rev. Lett. 109, 257203 (2012).

[15] M. J. Harris, S. T. Bramwell, D. F. McMorrow, T. Zeiske, and K. W. Godfrey, Phys. Rev. Lett. 79, 2554 (1997).

[16] R. Siddharthan, B. S. Shastry, A. P. Ramirez, A. Hayashi, R. J. Cava, and S. Rosenkranz, Phys. Rev. Lett. 83, 1854 (1999).

[17] B. C. den Hertog and M. J. P. Gingras, Phys. Rev. Lett. 84, 3430 (2000).

[18] C. Lobban, J. L. Finney, and W. F. Kuhs, J. Chem. Phys. 112, 7169 (2000).

[19] C. Salzmann, P. Radaelli, A. Hallbrucker, E. Mayer, and J. Finney, Science 311, 1758 (2006).

[20] Z.-Y. Chen and M. Kardar, J. Phys. C 19, 6825 (1986).

[21] L. Gu, B. Chakraborty, P. L. Garrido, M. Phani, and J. L. Lebowitz, Phys. Rev. B 53, 11985 (1996).

[22] S. Singamaneni and V. V. Tsukruk, Soft Matter 6, 5681 (2010).

[23] M. Gregory, Elastic Instability: Analysis of Buckling Modes and Loads of Framed Structures (Taylor and Francis, London, 1967).

[24] See Supplemental Material at http://link.aps.org/ supplemental/10.1103/PhysRevLett.112.098701 for details on analysis and experiments.

[25] R. Liebmann, Statistical Mechanics of Periodic Frustrated Ising Systems (Springer-Verlag, Berlin, 1986), pp. 77-81.

[26] A. S. Wills, R. Ballou, and C. Lacroix, Phys. Rev. B 66, 144407 (2002).

[27] S. P. Timoshenko and J. M. Gere, Theory of Elastic Stability (Courier Dover Publications, New York, 2012). 